\title{
Gases invernadero en aguas con bajo oxígeno en el reservorio eutrófico de Prado (Colombia)
}

\author{
Maribeb Castro-González*, Victoria Torres-Valdés \\ Laboratorio de Ecología Microbiana, Facultad de Ciencias, Universidad del Tolima, Ibagué, Colombia
}

\begin{abstract}
Resumen
En esta investigación se cuantificó la concentración de gases invernadero (metano y óxido nitroso) y de otros parámetros fisicoquímicos en la columna de agua del reservorio eutrófico de Prado para determinar con experimentos in situ el origen del óxido nitroso y la distribución vertical de ambos gases. Los resultados indicaron que la nitrificación contribuyó más activamente que la desnitrificación a la producción de óxido nitroso en la columna de agua, con tasas estimadas en 122,5 $\pm 79,9 \mathrm{nM} / \mathrm{d}$, y que comparado con otras hidroeléctricas y lagos en el mundo, las concentraciones de óxido nitroso (de hasta $38,6 \pm 0,9 \mathrm{nM}$ ) y de metano (de hasta $242 \pm 85,5 \mu \mathrm{M}$ ) cuantificadas en el embalse fueron altas. Este es el primer estudio desarrollado en la represa de Prado que muestra la sobresaturación de ambos gases en la columna de agua, lo que sugiere que esta hidroeléctrica podría estar emitiendo cantidades significativas de gases invernadero a la atmósfera.
\end{abstract}

Palabras clave: hidroeléctrica, embalse tropical, óxido nitroso, metano, nitrificación, desnitrificación.

Greenhouse gases in low-oxygen waters in the eutrophic reservoir of Prado (Colombia)

\begin{abstract}
In this study we quantified greenhouse gases concentration (nitrous oxide and methane) and other physicochemical parameters in the water column of Prado eutrophic reservoir to determine with in situ experiments the nitrous oxide source and the vertical distribution of both gases. The results indicated that nitrification contributed more actively than denitrification to nitrous oxide production in the water column to estimated rates of $122.5 \pm 79.9 \mathrm{nM} / \mathrm{d}$, and that, in comparison with other dams and lakes in the world, the nitrous oxide (up to $38.6 \pm 0.9 \mathrm{nM}$ ) and methane concentrations (up to $242 \pm 85.5 \mu \mathrm{M}$ ) measured in the reservoir were high. This is the first study in the Prado dam showing the oversaturation of both gases in the water column, which suggests that this reservoir could be emitting significant quantities of greenhouse gases to the atmosphere.
\end{abstract}

Key words: Dam, tropical reservoir, nitrous oxide, methane, nitrification, denitrification.

\section{Introducción}

En los últimos años los estudios sobre las emisiones de gases invernadero desde las hidroeléctricas han demostrado que esta fuente de energía no es limpia ni verde como se esperaba y que estos reservorios pueden emitir hasta 3,6 veces más gases invernadero que los generados por los combustibles fósiles (Fearnside, 2005). Entre estos gases se encuentra el óxido nitroso $\left(\mathrm{N}_{2} \mathrm{O}\right)$, con un potencial de calentamiento 310 veces mayor que el del $\mathrm{CO}_{2}$, una concentración en la tropósfera de 325 ppm y una contribución al calentamiento global del planeta de 5 a 7 \% (Intergovernmental Panel on Climate Change - IPCC, 2011). El segundo gas invernadero en orden de importancia es el metano, con un potencial de calentamiento 25 veces mayor que el del $\mathrm{CO}_{2}$ y cuya concentración en la atmósfera (1.819 ppbv) ha triplicado los niveles de la era preindustrial (IPCC, 2011). Ambos gases se generan en ecosistemas acuáticos, y en el caso del metano se estima que un $25 \%$ de sus emisiones son antropogénicas y un $69 \%$ son de origen microbiano (Borrel, et al., 2011, Demarty \& Bastien, 2011); de ellas 6 a $16 \%$ son emisiones provenientes de lagos, ríos y reservorios, estos últimos con emisiones estimadas de aproximadamente $3 \mathrm{Tg} \mathrm{C}-\mathrm{CH}_{4}$ (Barros, et al., 2011), producto de la metanogénesis en sedimentos y en la columna de agua anóxica (Borrel, et al., 2011, Sturm, et al., 2013). Por su parte, el $\mathrm{N}_{2} \mathrm{O}$ se deriva de procesos antropogénicos (procesos industriales y agrícolas) y biogénicos en ambientes terrestres, marinos y de aguas dulces (United States Environmental Protection Agency-EPA, 2010). Mediante estudios isotópicos se ha estimado que la contribución a las emisiones globales de los ambientes de agua dulce es de $24 \%$ (Snider, et al.,

\footnotetext{
*Correspondencia:

Maribeb Castro-González, mcastro@ut.edu.co

Recibido: 12 de mayo de 2015

Aceptado: 6 de agosto de 2015
} 
2015), y que los embalses tropicales aportan entre 130 y 6.9 $\mu \mathrm{g}$ de $\mathrm{N}_{2} \mathrm{O} / \mathrm{m} / \mathrm{d}$ (Guérin, et al., 2008, Yang, et al., 2014) debido a los procesos de nitrificación y desnitrificación, los cuales contribuyen con un $89 \%(\sim 16 \mathrm{Tg} / \mathrm{N} / \mathrm{año})$ a las emisiones anuales de $\mathrm{N}_{2} \mathrm{O}$ (Loescher, et al., 2012, Stocker, et al., 2013). En la nitrificación el $\mathrm{N}_{2} \mathrm{O}$ es un producto secundario de la oxidación de amonio a nitrito por la acción de bacterias y arqueobacterias (Casciotti \& Buchwald, 2012, Hatdzenpichler, 2012, Santoro, et al., 2011). En dicha oxidación se distinguen dos fuentes de este gas: la primera producida por la descomposición química de la hidroxilamina, y la segunda, por la reducción de nitrito en condiciones de bajos niveles de oxígeno $\left(\mathrm{O}_{2}\right)$, proceso también denominado desnitrificación nitrificante (Casciotti \& Buchwald, 2012). En la desnitrificación, el $\mathrm{N}_{2} \mathrm{O}$ es un producto intermedio de la reducción del óxido nítrico en niveles bajos de $\mathrm{O}_{2}$, por lo que puede darse acumulación de $\mathrm{N}_{2} \mathrm{O}$ si su reducción a nitrógeno atmosférico $\left(\mathrm{N}_{2}\right)$ se inhibe por la presencia de $\mathrm{O}_{2}$, lo que se conoce como desnitrificación parcial.

Aunque desde 1990 se ha venido observando que las hidroeléctricas contribuyen de manera significativa a la remoción del nitrógeno y a la emisión de gases invernadero (Rudd, et al., 1993, Harrison, et al., 2009), lo cual afecta el equilibrio global del $\mathrm{N}_{2} \mathrm{O}$ (St. Louis, et al., 2000), pues este se produce tanto en el epilimnio en condiciones óxicas como en el hipolimnio bajo condiciones subóxicas o anóxicas, son pocos los estudios que se han hecho en hidroeléctricas (24 de 741) en áreas tropicales (Goldenfum, 2012), a pesar de que su número ha aumentado en la última década alrededor del mundo y que las alteraciones hidrológicas asociadas con su construcción han alterado el ciclo de agua terrestre (Liu, et al., 2011).

Teniendo en cuenta estos antecedentes y dado que, a pesar de ser una de las más grandes e importantes de Colombia, en la represa de Prado en el sur del Tolima no se habían hecho estudios en este sentido, esta investigación se propuso analizar en muestras tomadas en campo la distribución de $\mathrm{N}_{2} \mathrm{O}, \mathrm{CH}_{4}$, nutrientes y otros parámetros físicoquímicos en la columna de agua, así como determinar en el laboratorio qué procesos microbianos estaban involucrados en la producción de $\mathrm{N}_{2} \mathrm{O}$ en el embalse.

\section{Materiales y métodos}

\section{Descripción del área de estudio}

La represa de Prado, una de las más grandes $\left(39 \mathrm{~km}^{2}\right)$ y antiguas de Colombia (42 años), está ubicada al suroeste del departamento de Tolima. Tiene un espejo de agua de 3.900 hectáreas, una profundidad promedio de $45 \mathrm{~m}$, estratificación térmica y química y fuerte eutrofización (Roldán, 2003). Las muestras de agua para el estudio se recolectaron en septiembre de 2012 (estación seca) en tres áreas del embalse cercanas a las veredas de Tomogó
(0343’37,2” N, 7453’51,0” O), Isla del Sol (0345’58,1” N, 7451'27,7”' O) y Lozanía (0352'19,18” N, 7448'16,5”, O) (Figura 1).

\section{Métodos de muestreo en campo}

En cada estación se hizo un perfil de oxígeno disuelto, $\mathrm{pH}$ y temperatura en la columna de agua $(0-10 \mathrm{~m}$ de profundidad) con un equipo multiparámetro marca Oakton ${ }^{\circledR}$. Las muestras de agua se tomaron con botellas Van Dorn cada dos metros de profundidad para obtener los perfiles verticales de gases $\left(\mathrm{N}_{2} \mathrm{O}\right.$ y $\left.\mathrm{CH}_{4}\right)$ y de nutrientes $\left(\mathrm{NH}_{4}^{+}, \mathrm{NO}_{3}^{-}{ }^{-} \mathrm{y} \mathrm{NO}_{2}^{-}\right)$.

Las muestras de agua para determinar las cantidades de $\mathrm{N}_{2} \mathrm{O}$ y $\mathrm{CH}_{4}$ se pasaron directamente de las botellas de muestreo a tubos Labco Exetainer de tapa rosca de $11 \mathrm{ml}$, especiales para la toma de gases cuidando de eliminar todas las burbujas de aire durante el llenado por reflujo. Luego se agregaron $50 \mu \mathrm{l}$ de solución saturada de $\mathrm{HgCl}_{2}$ en cada tubo y se refrigeraron las muestras boca abajo a $4{ }^{\circ} \mathrm{C}$ hasta el momento de cuantificarlas en el laboratorio.

Para la determinación de nutrientes, se filtraron $500 \mathrm{ml}$ de las muestras de agua sobre membranas de $0.22 \mu \mathrm{M}$ (Millipore, Durapore), y se congelaron a $-20{ }^{\circ} \mathrm{C}$ hasta $\mathrm{su}$ cuantificación.

Para realizar los experimentos de producción de $\mathrm{N}_{2} \mathrm{O}$, se llenaron tres de las botellas con agua tomada a $4 \mathrm{~m}$ de profundidad en la estación Tomogó y se refrigeraron bajo oscuridad hasta el desarrollo de los experimentos en el laboratorio.

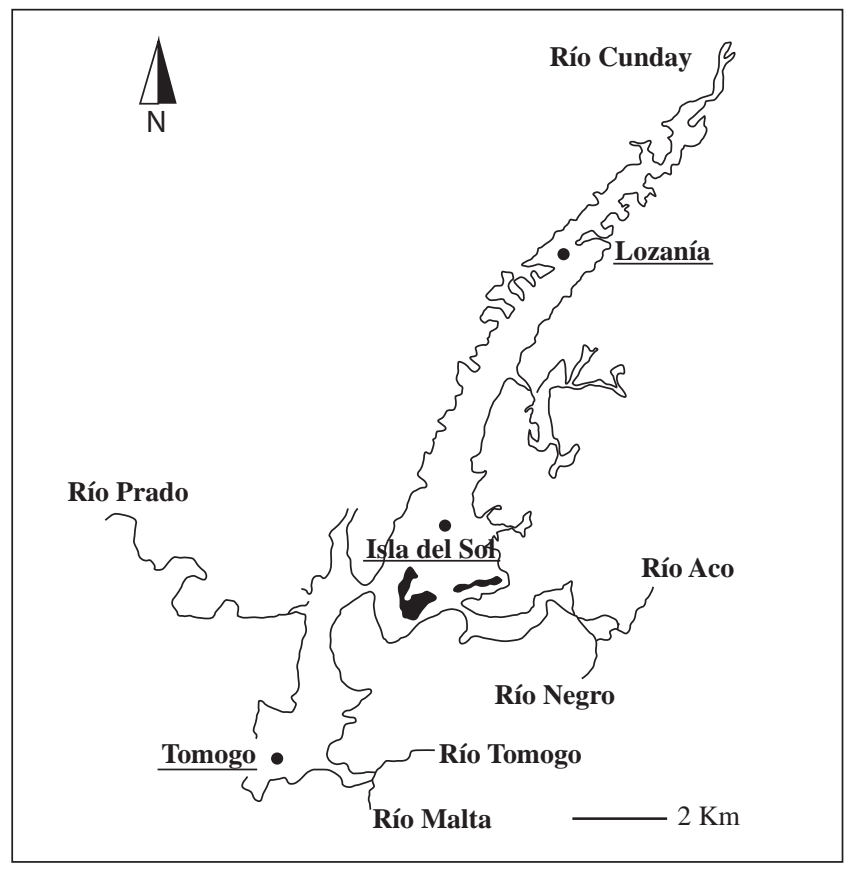

Figura 1. Ubicación de las estaciones de muestreo en el embalse de Prado, Tolima. (Modificada de Guevara, et al., 2009) 


\section{Experimentos de laboratorio y cuantificación de gases y nutrientes}

Estimación de la producción de $\mathrm{N}_{2} \mathrm{O}$ en muestras de agua. Los experimentos se hicieron bajo atmosfera de nitrógeno, con el fin de evitar la oxigenación de las muestras de agua tomadas en campo. A cada una de las tres botellas (1 1itro) se le agregó cloranfenicol (1g/l) para evitar la síntesis enzimática durante el desarrollo de los ensayos. Una botella se usó para estimar la producción neta de $\mathrm{N}_{2} \mathrm{O}$ (botella de control), y las otras dos botellas se utilizaron para estimar la producción de $\mathrm{N}_{2} \mathrm{O}$ por nitrificación agregando el inhibidor aliltiourea (ATU) en una y acetileno en la otra.

Estimación de la producción neta de $\mathrm{N}_{2} \mathrm{O}$. La producción neta de $\mathrm{N}_{2} \mathrm{O}$ se estimó como la producción de este gas por desnitrificación y por nitrificación en los ensayos control. Para ello, las muestras de agua (1 litro) con cloranfenicol se distribuyeron en frascos de $60 \mathrm{ml}$ que se sellaron con gomas de butilo y tapas metálicas. Posteriormente, los frascos se incubaron durante 24 horas en la oscuridad y a temperatura ambiente $\left(23^{\circ} \mathrm{C}\right)$ y cada seis horas $(0,6,12,24 \mathrm{~h})$ se tomaban tres de ellos y se les añadían $100 \mu \mathrm{lde} \mathrm{HgCl}_{2}$, y se refrigeraban a $4{ }^{\circ} \mathrm{C}$ hasta el momento de cuantificar el $\mathrm{N}_{2} \mathrm{O}$ en la fase gaseosa de cada uno.

Estimación de la producción de $\mathrm{N}_{2} \mathrm{O}$ por nitrificación. Para este ensayo, a una de las botellas se le agregó $1 \mathrm{~g} / 1$ de ATU, la cual se conoce como inhibidor de la oxidación de amonio a nitrito (Ginestet, et al., 1998), evitando así la producción de $\mathrm{N}_{2} \mathrm{O}$ por nitrificación. Posteriormente, esta muestra de agua con ATU se distribuyó en frascos de suero de $60 \mathrm{ml}$ que se sellaron con gomas de butilo y tapas metálicas. Simultáneamente se desarrolló otro ensayo en el cual se inyectó suavemente a través de la goma de butilo $15 \% \mathrm{v} / \mathrm{v}$ de acetileno en los frascos de $60 \mathrm{ml}$ sellados herméticamente en los cuales se habían mantenido las muestras de agua, para lo cual se tuvo en cuenta que también se ha reportado su acción inhibitoria sobre la amonio monooxigenasa (Wrage, et al., 2004). A continuación, los frascos de ambos tratamientos se incubaron y se manipularon bajo las condiciones ya indicadas para cuantificar el $\mathrm{N}_{2} \mathrm{O}$ en la fase gaseosa. La producción de $\mathrm{N}_{2} \mathrm{O}$ por nitrificación se estimó con base en la diferencia entre las tasas cuantificadas en los experimentos de producción neta de $\mathrm{N}_{2} \mathrm{O}$ y las tasas cuantificadas en los experimentos con ATU o con acetileno.

Cuantificación de nitrógeno amoniacal, nitritos y nitratos. La determinación de nitrógeno amoniacal se hizo mediante el método de Kjeldahl y la de los nitratos y nitritos mediante lecturas espectrofotométricas a $220-275 \mathrm{~nm}$ y $510 \mathrm{~nm}$, respectivamente, según los métodos descritos para el análisis de nutrientes en aguas en el manual Standard Methods for the Examination of Water and Wastewater (1999).

Cuantificación de $\mathrm{N}_{2} \mathrm{O}$ y $\mathrm{CH}_{4}$ en las muestras de agua y en los experimentos. Para esto se utilizó la técnica de equilibrio de la fase gaseosa y líquida descrita por McAuliffe (1971): se inyectó suavemente $1 \mathrm{ml}$ de helio ultrapuro en los tubos de $11 \mathrm{ml}$ correspondientes a los perfiles verticales, y $10 \mathrm{ml}$ en los frascos de los experimentos (60 ml). Posteriormente, se incubaron durante una hora a $40{ }^{\circ} \mathrm{C}$ con agitación para generar el equilibrio de gases entre ambas fases. A continuación, se tomó una muestra de la fase gaseosa $(1 \mathrm{ml}$ de los tubos y $10 \mathrm{ml}$ de los frascos) y se inyectó en viales evacuados para su cuantificación con un cromatógrafo de gases (Shimadzu GC), equipado con un detector de captura de electrones ${ }^{63} \mathrm{Ni}$ a $350{ }^{\circ} \mathrm{C}$, con fase móvil de nitrógeno de alta pureza. Se usaron los estándares Scotty de metano y óxido nitroso balanceados en nitrógeno con una precisión de $\pm 5 \%$. La concentración de $\mathrm{N}_{2} \mathrm{O}$ disuelto se calculó usando la fórmula de solubilidad de Weiss \& Price (1980), y la concentración de $\mathrm{CH}_{4}$ disuelto, con los datos de solubilidad de Wiesenburg \& Guinasso (1979).

\section{Análisis estadístico}

Para estimar las tasas de producción y consumo de óxido nitroso $(\mathrm{nM} / \mathrm{d})$ se hizo un análisis de regresión lineal con los datos obtenidos durante las 24 horas del experimento, y la tasa se estimó a partir de la pendiente obtenida en dicho análisis. Los valores positivos indicaron producción o acumulación de $\mathrm{N}_{2} \mathrm{O}$ y los valores negativos representaron su consumo.

\section{Resultados}

\section{Caracterización fisicoquímica de la columna de agua}

Distribución de oxígeno, pH y temperatura. En general, se registró una fuerte oxiclina en las tres áreas de estudio. En Tomogó los niveles de oxígeno descendieron desde $151 \mu \mathrm{M}$ en la superficie hasta $34 \mu \mathrm{M}$ a los 7 metros de profundidad. Entre los 7 y 10 metros de profundidad la concentración de $\mathrm{O}_{2}$ se mantuvo más o menos igual, excepto por un leve descenso $\left(31,2 \mu \mathrm{M} \mathrm{O}_{2}\right)$ detectado a 9 metros. En el área de Isla del Sol, la disminución de $\mathrm{O}_{2}$ fue levemente mayor que en Tomogó, pues partió de $122 \mu \mathrm{M}$ en la superficie hasta llegar a los $24 \mu \mathrm{M}$ a los 10 metros de profundidad. Sin embargo, fue en el área de Lozanía donde se registró la mayor variación de oxígeno en la columna de agua, con niveles superficiales de $93 \mu \mathrm{M} \mathrm{O}_{2}$ (54\% de saturación), niveles de hipoxia $\left(<62 \mu \mathrm{M} \mathrm{O}_{2}\right)$ entre los 2 y los 4 metros, de suboxia $\left(<4 \mu \mathrm{M} \mathrm{O}_{2}\right)$ entre los 5 y los 6 metros y de anoxia entre los 7 y los 10 metros de profundidad.

En las estaciones de muestreo el pH fluctuó de 9 a 6 entre la superficie y el fondo. La estación que presentó el mayor rango de $\mathrm{pH}$ entre superficie y fondo fue Tomogó ( 8,9 y 6,3), y la que presentó el rango más estrecho en el perfil vertical fue Lozanía (entre 7 y 6,2). La temperatura en la columna de agua de las tres estaciones fluctuó entre los $29^{\circ} \mathrm{C}$ en la superficie y los $25^{\circ} \mathrm{C}$ en el fondo. En la estación Lozanía se observó un rango de temperatura más estable $\left( \pm 1^{\circ} \mathrm{C}\right)$ en el perfil vertical con respecto a lo observado en las otras dos estaciones, donde el rango de variación entre la superficie y el fondo fue más amplio $\left(3-4{ }^{\circ} \mathrm{C}\right)$ (Figura 2). 


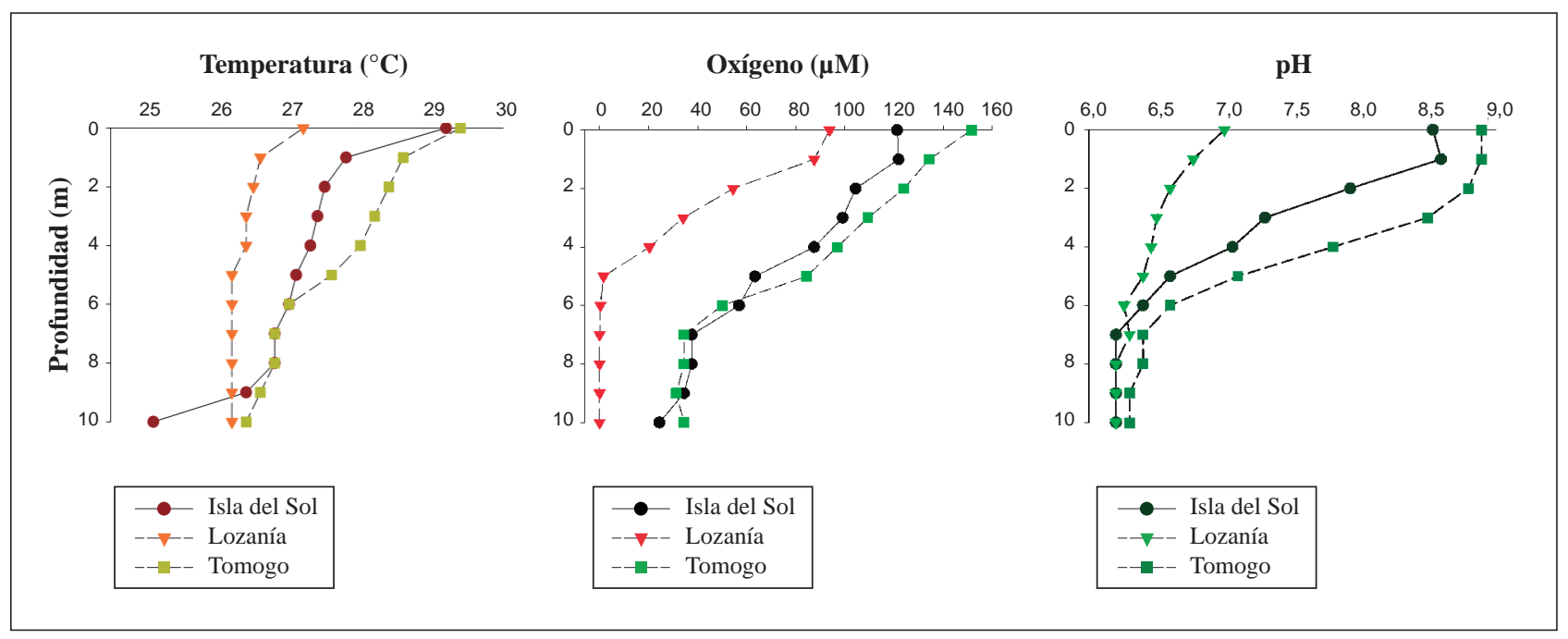

Figura 2. Perfiles verticales de oxígeno, $\mathrm{pH}$ y temperatura en las estaciones muestreadas en el embalse de Prado

Distribución de los nutrientes disueltos en la columna de agua. El amonio fue el nutriente que presentó mayor variabilidad y concentración en la columna de agua del embalse de Prado, con valores mínimos de 2,8 $\mu \mathrm{M}$, y un valor máximo de $50 \mu \mathrm{M}$ cuantificados en Lozanía a los $8 \mathrm{~m}$ de profundidad; allí mismo se detectó anoxia y bajos niveles de $\mathrm{pH}(6,2)$. En la Isla del Sol se observó un incremento de amonio de 10 a $35 \mu \mathrm{M}$ desde los 2 hasta los 8 metros de profundidad (con 37,5 $\mu \mathrm{M}$ de $\mathrm{O}_{2}$ ), y un consumo subsiguiente hasta los $10 \mathrm{~m}$. En Tomogó, por el contrario, se observó una disminución en los niveles de amonio de 18 a $3 \mu \mathrm{M}$ entre $\operatorname{los} 2$ y los 6 metros de profundidad, con dos picos de producción: el primero a los 2 metros $(18 \mu \mathrm{M})$ y el otro a los 8 metros $(21 \mu \mathrm{M})$, con un nivel de $\mathrm{O}_{2}$ de $34,4 \mu \mathrm{M}$. Los mismos picos de producción se detectaron un año después en la misma época de muestreo a los 3 y los 7 metros de profundidad en concentraciones de 30 y $50 \mu \mathrm{M}$, respectivamente (datos no mostrados). En Lozanía también se presentaron dos picos de producción de amonio, uno a los 4 metros $(12 \mu \mathrm{M})$ y otro a los 8 metros $(50,4 \mu \mathrm{M})$, siendo este último el valor máximo de amonio reportado para el área de estudio.

En general, en la columna de agua de las estaciones muestreadas las concentraciones de nitratos fueron bajas $(2,6-5 \mu \mathrm{M})$. Sin embargo, fueron evidentes dos picos de producción: uno a los 8 metros en Isla del Sol $(12,6 \mu \mathrm{M})$ y otro en Tomogó a los 4 metros $(36,1 \mu \mathrm{M})$, lo que indica procesos de nitrificación en ambas profundidades con niveles de oxígeno de 37,5 $\mu \mathrm{M}$ y $97 \mu \mathrm{M}$, respectivamente. Los niveles de nitrito fueron muy bajos en las tres estaciones de muestreo $(1-1,7 \mu \mathrm{M})$ entre los 0 y los 10 metros de profundidad (Figura 3).

Distribución de gases invernadero. En la columna de agua del embalse de Prado el $\mathrm{N}_{2} \mathrm{O}$ disuelto fluctuó entre $26 \pm 7,3$ y 38,6 $\pm 0,9$ nM. Las concentraciones más bajas se cuantificaron en Isla del Sol (entre $26 \pm 7,34$ y 33,2 \pm 3,4 nM), seguidas por las de Tomogó (entre 26,9 \pm 5,9 y 34,9 $\pm 6,4 \mathrm{nM}$ ) y las de Lozanía, en donde se reportaron los valores más altos (entre 30,9 $\pm 0,1$ y 38,6 \pm 0,9 nM) en las muestras recolectadas en época seca. En general, los datos del perfil vertical mostraron una producción de $\mathrm{N}_{2} \mathrm{O}$ hacia los 3 metros de profundidad, principalmente en la estación de Lozanía, seguida por Isla del Sol y Tomogó (en menor grado). Se evidenció, asimismo, consumo de $\mathrm{N}_{2} \mathrm{O}$ en la profundidad, siendo muy fuerte entre los 6 y $\operatorname{los} 7$ metros en las estaciones de Isla del Sol y Tomogó y un poco menos evidente en la estación de Lozanía entre los 5 y los 8 metros.

El porcentaje de saturación de $\mathrm{N}_{2} \mathrm{O}$ en la columna de agua del embalse de Prado osciló entre 222 y 330 \%, lo cual significa que se presentaba una alta sobresaturación de este gas invernadero en la represa. La sobresaturación fue similar en todas las estaciones de muestreo, sin embargo, la estación de Lozanía presentó valores por encima del 300 \% a lo largo de la columna de agua, con un máximo de $330 \%$ a 3 metros de profundidad, mientras que la estación de Isla del Sol mostró los menores rangos de saturación en el perfil vertical (222-283 \%).

En la columna de agua del embalse el $\mathrm{CH}_{4}$ disuelto osciló entre $0,2 \pm 0,05$ y $242 \pm 85,5 \mu \mathrm{M}$. Las concentraciones más bajas se cuantificaron en Isla del Sol (entre 0,25 $\pm 0,05 \mathrm{y}$ $72,7 \pm 24,4 \mu \mathrm{M})$, seguidas por las de Tomogó (0,37 $\pm 0,08$ y $80 \pm 4,0 \mu \mathrm{M})$ y las de Lozanía, en donde se reportaron los valores más altos (entre 0,3 $\pm 0,12$ y $242 \pm 85,5 \mu \mathrm{M}$ ). En Isla del Sol el metano disuelto se mantuvo uniforme a lo largo de la columna de agua $(0,2-0,6 \mu \mathrm{M})$ hasta los 8 metros y entre los 9 y los 10 metros su concentración incrementó de 15 a $72 \mu \mathrm{M}$. En Tomogó se detectaron dos picos de producción: el primero entre los 3 y los 4 metros $(1-1,7 \mu \mathrm{M})$ y el segundo a los 10 metros $(80 \mu \mathrm{M})$, en tanto que en Lozanía se detectó 
una alta concentración de metano a partir de los 4 metros $(11,4 \mu \mathrm{M})$, que se incrementó con la profundidad y alcanzó el máximo a los 10 metros $(242 \mu \mathrm{M})$.

Los resultados mostraron que el porcentaje de saturación de metano en la columna de agua del embalse de Prado en relación con las concentraciones medidas en la atmósfera durante el muestreo $(0,10 \mu \mathrm{M})$, osciló entre $225 \%$ hasta $235 \%$ (cuantificado a 10 metros de profundidad en la estación Lozanía), lo cual evidencia una alta sobresaturación de este gas invernadero en esta localidad. La estación de Isla del Sol mostró los menores rangos de saturación en el perfil vertical entre los 0 y los 8 metros (225 - 582\%), y la estación de Lozanía presentó los más altos valores de saturación (>11.000 \%) desde los 4 metros de profundidad hasta el fondo, lo que se asoció a la observación en campo de burbujas de metano que ascendían del fondo a la superficie (Figura 4).

\section{Experimentos de producción de óxido nitroso en la columna de agua}

Los resultados de los experimentos (Tabla 1) mostraron que en el área de Tomogó se produjo $\mathrm{N}_{2} \mathrm{O}$ a una tasa de 141,6 $\pm 91,46 \mathrm{nM} / \mathrm{d}$ por procesos tanto de nitrificación como de desnitrificación a los 4 metros de profundidad y bajo condiciones subóxicas $\left(3,5 \mathrm{mg} / \mathrm{l} \mathrm{O}_{2}\right)$.

Los experimentos con acetileno, por su parte, mostraron una fuerte inhibición de la producción de $\mathrm{N}_{2} \mathrm{O}$ por parte de la comunidad nitrificante (se inhibió la oxidasa de amonio de bacterias y arqueobacterias), lo que supuso observar en los ensayos consumo de óxido nitroso a tasas de 49,6 \pm 31 $\mathrm{nM} / \mathrm{d}$ sugiriendo una reducción de $\mathrm{N}_{2} \mathrm{O}$ a $\mathrm{N}_{2}$ por acción de la comunidad desnitrificante presente a esta profundidad.

En los experimentos con ATU se observó una inhibición de la producción de $\mathrm{N}_{2} \mathrm{O}$ por nitrificación, lo que llevó a que

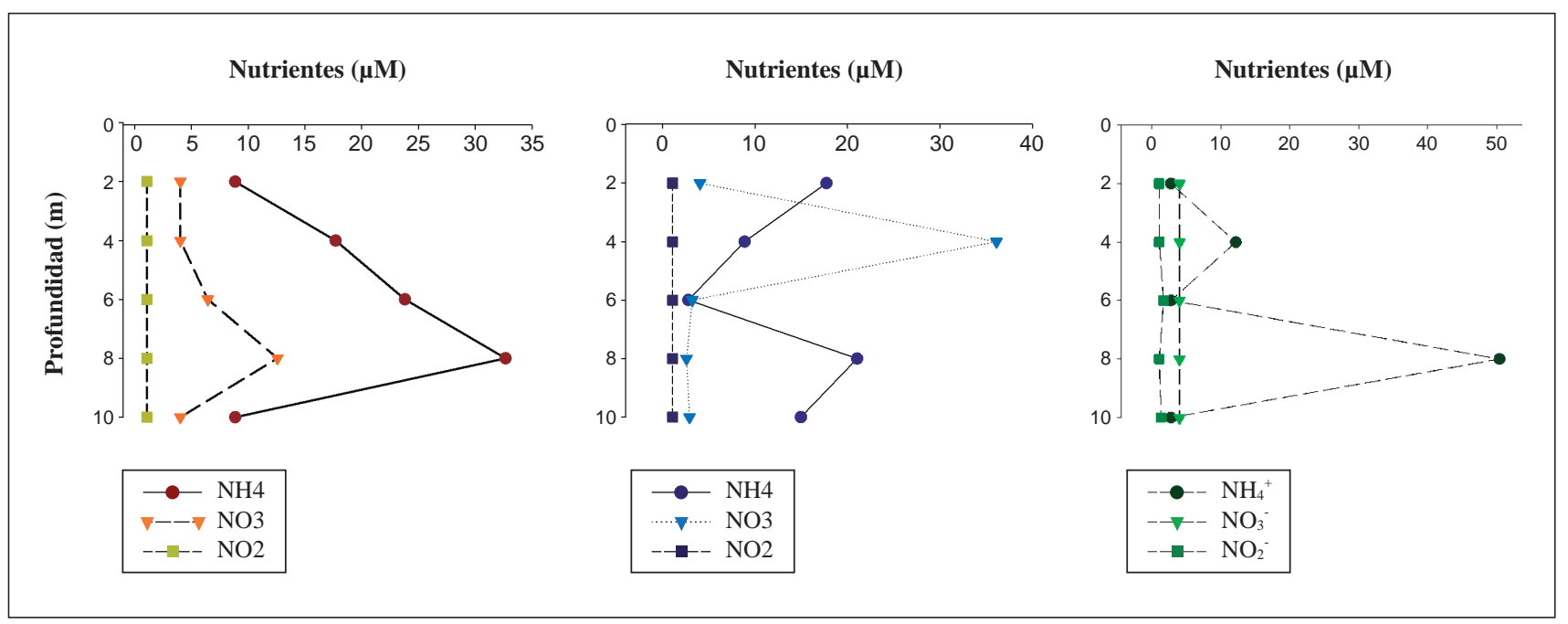

Figura 3. Perfil vertical de nutrientes (amonio, nitrato y nitrito) en las estaciones muestreadas en el embalse de Prado

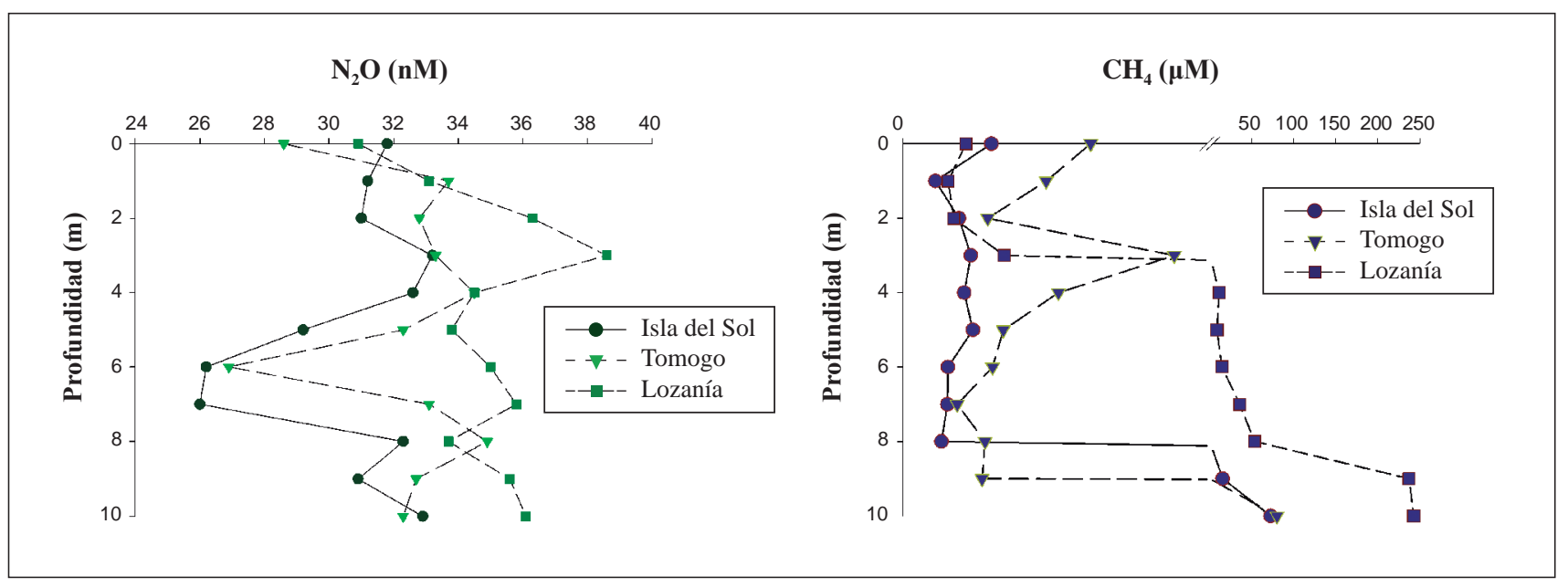

Figura 4. Perfil vertical de $\mathrm{N}_{2} \mathrm{O}$ y $\mathrm{CH}_{4}$ disuelto en la columna de agua de las estaciones muestreadas en el embalse de Prado 
Tabla 1. Tasas de producción de óxido nitroso en la estación de Tomogó, embalse de Prado

\begin{tabular}{lllc}
\hline Experimento & Proceso que se midió en cada experimento & nM/d \\
\hline Control & Producción neta de $\mathrm{N}_{2} \mathrm{O}$ tanto por nitrificación como por desnitrificación & $141,6 \pm 91,46$ \\
Con adición de acetileno & Inhibición del $\mathrm{N}_{2} \mathrm{O}$ producido por nitrificación y su probable consumo por desnitrificación & $-49,6 \pm 31,0$ \\
Con adición de aliltiourea & Inhibición de $\mathrm{N}_{2} \mathrm{O}$ producido por nitrificación y su probable producción por desnitrificación & $19,08 \pm 11,5$ \\
& $\begin{array}{l}\mathrm{N}_{2} \mathrm{O} \text { producido por nitrificación estimado a partir de la diferencia entre las tasas obtenidas en } \\
\text { el experimento control y las del experimento con aliltiourea }\end{array}$ & $122,5 \pm 79,9$ \\
\hline
\end{tabular}

la tasa de producción del gas bajara a niveles de 19,08 \pm $11,5 \mathrm{nM} / \mathrm{d}$. Muy probablemente esta tasa correspondía únicamente a la producción de $\mathrm{N}_{2} \mathrm{O}$ generada por las bacterias desnitrificantes presentes en la columna de agua que no fueron inhibidas por la ATU.

La diferencia entre la tasa estimada en los experimentos de control y la estimada en los experimentos con ATU permitió determinar que en Tomogó se estaba produciendo $\mathrm{N}_{2} \mathrm{O}$ por nitrificación a tasas de 122,5 $\pm 79,9 \mathrm{nM} / \mathrm{d}$.

\section{Discusión}

Los perfiles verticales de temperatura, oxígeno y nutrientes hallados en este estudio coinciden con estudios previos que mostraban la estratificación térmica y química del embalse (Márquez \& Guillot, 1988). El pH básico de entre 8,5 y 8,9 en la superficie de las estaciones de Tomogó e Isla del Sol registrado en este estudio difiere de los valores de 6,1 a 7,4 reportados previamente (Roldán, 2003). Por otro lado, en este estudio se observó que los niveles de $\mathrm{pH}$, nitrato y oxígeno en la columna de agua en la estación de Lozanía fueron más bajos que los reportados (Márquez \& Guillot, 1988, Roldán, 2003), lo que indica que en la actualidad la anoxia tiende a ser más somera a partir de los 7 metros de profundidad.

Tanto los perfiles verticales de oxígeno como los de nutrientes permiten sugerir que varios procesos microbianos están teniendo lugar en el área de estudio, lo que respalda las hipótesis planteadas anteriormente con respecto a la distribución de formas nitrogenadas en los embalses eutróficos (Roldán, 2003). Uno de ellos es la amonificación que se observó al cuantificar altas concentraciones de amonio tanto en aguas superficiales, entre los 2 y los 4 metros de profundidad $(35 \mu \mathrm{M})$, como en aguas un poco más profundas, a los 8 metros $(50 \mu \mathrm{M})$. En el primer caso, probablemente la excreción de amonio por parte de los organismos y los procesos de remineralización de la materia orgánica contribuyen a los altos niveles de este compuesto encontrados en el epilimnio. Teniendo en cuenta los bajos niveles de oxígeno $(<40 \mu \mathrm{M})$ en la profundidad $(8 \mathrm{~m})$, es probable que la elevada producción de amonio sea producto del proceso de reducción desasimilatoria del nitrato, sobre todo en la estación de Lozanía, donde los niveles de nitrato y de oxígeno estuvieron en el límite de detección en toda la columna de agua. Este proceso se ha observado con frecuencia en otros ecosistemas acuáticos con niveles limitados de nitrato y ricos en carbono orgánico (Bruner \& García-Gil, 1996, Kelso, et al., 1997, Burgin \& Hamilton, 2007, Figueiredo, et al., 2012), sin embargo, para corroborar esta hipótesis es necesario adelantar estudios en el área que permitan confirmar si se está dando dicha reacción.

Los resultados también sugieren que la nitrificación y desnitrificación tienen lugar en la columna de agua. En el primer caso, la oxidación de amonio a nitrato podría explicar los dos picos de producción de nitrato observados a 4 metros en Tomogó $(36,1 \mu \mathrm{M})$ bajo condiciones óxicas y a 8 metros en Isla del Sol $(12,6 \mu \mathrm{M})$ bajo condiciones hipóxicas. Esta observación se corroboró indirectamente en Tomogó al observar los altos niveles de $\mathrm{N}_{2} \mathrm{O}(34,5 \pm 1,2 \mathrm{nM})$ en los perfiles verticales y los resultados de los experimentos que indicaron que la nitrificación contribuyó en mayor medida a la producción de $\mathrm{N}_{2} \mathrm{O}$, dado que este gas es un producto obligado de la oxidación de amonio a nitrito causada por bacterias y arqueobacterias (Hiorns, et al., 1995, Hastings, et al., 1998, Speksnijder, et al., 1998, Santoro, et al., 2011).

En el caso de la desnitrificación, esta también parece darse en la columna de agua, ya que se observaron bajos niveles de nitrato en todas las estaciones (entre los 2 y los 6 metros en Isla del Sol, entre los 6 y los 10 metros en Tomogó y en toda la columna de agua en Lozanía), a lo que se suma la detección de una leve reducción del $\mathrm{N}_{2} \mathrm{O}$ entre los 4 y $\operatorname{los} 8$ metros de profundidad en las estaciones de Tomogó e Isla del Sol a medida que el oxígeno disminuía. Esto sugiere que se produjo la reducción del $\mathrm{N}_{2} \mathrm{O}$ a $\mathrm{N}_{2}$ por desnitrificación, evidenciada, además, en los experimentos llevados a cabo con las muestras de agua de Tomogó, en los cuales hubo consumo de $\mathrm{N}_{2} \mathrm{O}(-49,6 \pm 31,0 \mathrm{nM} / \mathrm{d})$ durante la incubación, probablemente por la acción de bacterias desnitrificantes.

Además, los resultados de los perfiles verticales de $\mathrm{N}_{2} \mathrm{O}$ y $\mathrm{CH}_{4}$ indican que varios procesos microbianos podrían explicar los altos niveles de saturación de ambos gases de efecto invernadero cuantificados en el área. Para el caso del $\mathrm{N}_{2} \mathrm{O}$ se observó una alta concentración y saturación en los perfiles verticales, lo que concuerda con los datos reportados en lagos eutróficos (hasta 557 \%) (Wang, et al., 2006, 2009, 2010), en estuarios subtropicales (hasta $435 \%$ ) (Musenze, et al., 2014), en embalses subtropicales en Australia (hasta 220 \%) (Sturm, et al., 2013), en reservorios chinos (entre 
201 y 689 \% (Liu, et al., 2011) y en el lago eutrófico Lacamas (hasta $750 \%$ ) (Deemer, et al., 2011). Dado que las aguas superficiales registran niveles elevados de saturación con respecto a las concentraciones de $\mathrm{N}_{2} \mathrm{O}$ atmosféricas en equilibrio (325 ppbv; IPCC, 2011), se puede decir que la represa de Prado es una fuente de emisiones de $\mathrm{N}_{2} \mathrm{O}$ a la atmósfera, con lo que se ratifica lo reportado por Guérin, et al. (2008), quienes indicaron que la contribución a las emisiones globales de gases invernadero de las emisiones netas de $\mathrm{N}_{2} \mathrm{O}$ de los reservorios tropicales puede ser del orden de $10 \%$.

Los altos niveles de saturación de $\mathrm{N}_{2} \mathrm{O}$ cuantificados en la columna de agua pueden explicarse por varios factores: primero, su producción in situ por bacterias de vida libre o aquellas asociadas al material en partículas, como se ha cuantificado en lagos mesotróficos (Abe, et al., 2000), y por nitrificantes en el epilimnio y desnitrificantes en el hipolimnio; en este último caso, se ha reportado una correlación negativa entre el $\mathrm{N}_{2} \mathrm{O}$ producido por desnitrificación y los niveles de oxígeno disuelto en aguas profundas o cercanas al sedimento (Liu, et al., 2011, Deemer, et al., 2011). Segundo, por la estratificación, que implica tiempos de residencia del agua estimados entre 50,3 y 136 días (Márquez \& Guillot, 2001, Roldán, 2003), evitando que los gases se mezclen y se difundan hacia la superficie. Tercero, por la liberación y transporte del $\mathrm{N}_{2} \mathrm{O}$ del sedimento hacia la superficie como lo han reportado Liu, et al. (2011). Cuarto, por oxidación aeróbica y anaeróbica del metano causada por bacterias y arqueobacterias, pues el $\mathrm{CH}_{4}$ se encontró en todas las estaciones y, específicamente en la estación de Lozanía, se observó que los niveles de $\mathrm{N}_{2} \mathrm{O}$ fueron los más altos y se mantuvieron más o menos estables a lo largo de la columna de agua y a pesar de la anoxia a partir de los 7 metros, lo que indica que, probablemente, la desnitrificación fue reemplazada por la oxidación del $\mathrm{CH}_{4}$. Sin embargo, aunque se ha reportado la producción de $\mathrm{N}_{2} \mathrm{O}$ mediante este proceso en lagos (Borrel, et al., 2011), es necesario verificar si dicha reacción se está dando en el embalse.

En el caso del $\mathrm{CH}_{4}$, los perfiles verticales indicaron claramente que había una gran producción de este gas por debajo de los 8 metros de profundidad en el embalse, probablemente por metanogénesis en los sedimentos y en la columna de agua anóxica, principalmente en el área de Lozanía, donde se alcanzaron concentraciones de hasta $242 \mu \mathrm{M}$, por lo que la columna de agua se mantiene sobresaturada, convirtiendo la represa en una importante fuente de emisión del gas hacia la atmósfera, como fue evidente por el burbujeo o ebullición del $\mathrm{CH}_{4}$ propia de este gas en la superficie. Actualmente se estima que entre 6 y $16 \%$ de las emisiones naturales de $\mathrm{CH}_{4}$ provienen de los lagos y ríos; en ese sentido, los datos aquí registrados concuerdan con lo reportado por Palma-Silva, et al., (2013) en otros lagos en los cuales se registró claramente el proceso de metanogénesis en los sedimentos (con producciones de hasta $1.727 \mu \mathrm{mol} / \mathrm{g}$ ) y en la columna de agua (hasta 19,9 $\mu \mathrm{mol} / \mathrm{l}$ ), así como en la columna de agua anóxica (Borrel, et al., 2011, Furlanetto, et al., 2012), e incluso en la columna de agua oxigenada de algunos lagos (Grossart, et al., 2011). En general, los valores de saturación superficial encontrados en Prado (420-1.200 \%) estaban en el mismo rango reportado para otras hidroeléctricas, con valores de hasta $600 \mu \mathrm{M}$ (Sturm, et al., 2013, Soumis, et al., 2004), aunque por debajo de lo reportado en la superficie de lagos someros (entre $2.000 \mathrm{y}$ $8.000 \%$ ) por Wang, et al., (2010).

Si bien los resultados de sobresaturación en los primeros 10 metros de la columna de agua indican que después de 40 años de construida, la represa de Prado es una fuente de gases invernadero (metano y óxido nitroso), hay que tener muy en cuenta que para estimar acertadamente las emisiones y los flujos totales de ambos gases desde cualquier ecosistema acuático debe recopilarse información de otros factores ambientales además de los analizados en esta investigación (nivel de oxígeno, temperatura), como son la competencia por sustratos o aceptores de electrones, la calidad y cantidad de la materia orgánica (Borrel, et al., 2011), el tiempo de residencia del agua, la edad y la forma del reservorio, la velocidad y la dirección del viento, la biomasa de organismos planctónicos y bentónicos, la concentración de carbono y nitrógeno inorgánico disuelto, la variación diaria en las concentraciones de gases y la producción neta de ambos gases comparando la producción antes y después de la construcción de la hidroeléctrica (Goldenfum, 2012, Wang, et al., 2010).

Los resultados de este estudio indican, por otra parte, que en el área de Tomogó se está dando un acople en la producción de $\mathrm{N}_{2} \mathrm{O}$, tanto por procesos de nitrificación como de desnitrificación, a bajos niveles de oxígeno $\left(109 \mu \mathrm{M}\right.$ de $\left.\mathrm{O}_{2}\right)$ y a 4 metros de profundidad. La nitrificación fue el proceso que más contribuyó a la producción de este gas invernadero, con tasas de 122,5 nM/d, mientras que la desnitrificación lo hizo a tasas de $19 \mathrm{nM} / \mathrm{d}$. Dichos resultados concuerdan con reportes previos sobre la mayor contribución de la nitrificación a la producción de $\mathrm{N}_{2} \mathrm{O}$ en el hipolimnion del lago Lacamas (Deemer, et al., 2011), en el lago Nakaumi (Senga, et al., 2002) y en dos hidroeléctricas en China (Liu, et al., 2011), en tanto que la desnitrificación se ha reportado como fuente de $\mathrm{N}_{2} \mathrm{O}$ en la columna de agua del lago Taihu (China) en relación con bajos niveles de oxígeno y nitrato (Wang, et al., 2010). La tasa estimada de producción de $\mathrm{N}_{2} \mathrm{O}$ por desnitrificación en este estudio concuerda con lo reportado en el hipolimnio del lago Kizaki (hipóxico y mesotrófico), en donde se han cuantificado entre 11 y 240 nM de $\mathrm{N}_{2} \mathrm{O}$ al día (Abe, et al., 2000), tasas estas más bajas que las tasas potenciales $\left(38,2 \mathrm{nM}\right.$ de $\mathrm{N}_{2} \mathrm{O}$ al día) reportadas en el hipolimnio del reservorio meromíctico Lacamas (Deemer, et al., 2011). Estos datos corroboran lo hallado en estudios previos en el área, que evidenciaron la presencia de bacterias desnitrificantes de tipo nos $Z$ en la columna de agua 
óxica e hipóxica de Isla del Sol (Castro-González, 2014), y la riqueza y diversidad de las bacterias que pueden reducir el $\mathrm{N}_{2} \mathrm{O}$ a $\mathrm{N}_{2}$ en el agua de bajo oxígeno de Tomogó y Lozanía (Castro-González \& Pacheco, 2014).

Aunque a partir de los experimentos se estimó la tasa de producción de $\mathrm{N}_{2} \mathrm{O}$ por nitrificación y desnitrificación, es importante resaltar algunas observaciones que se hicieron durante los ensayos con respecto al efecto de los inhibidores. En primer lugar, en relación con el efecto de la ATU, Jensen, et al., (2007) han reportado que en una concentración final de $886 \mu \mathrm{M}$ en sedimentos, puede inhibir en un $20 \%$ la desnitrificación y la oxidación anaerobia del ion amonio (anamox) e, incluso, producir la inhibición de las bacterias metanótrofas (Deutzman \& Schink, 2011), por lo cual la disminución en la producción de $\mathrm{N}_{2} \mathrm{O}$ detectada durante los experimentos con inhibidores puede afectar el acople entre los diferentes microrganismos presentes en la columna de agua. En este caso fue evidente que la inhibición producida por la ATU en el consumo de $\mathrm{N}_{2} \mathrm{O}$ alcanzó $75 \%$, lo que sugiere un efecto significativo sobre la comunidad responsable de este último paso de la reducción a $\mathrm{N}_{2}, \mathrm{o}$, quizá, mediante la estimulación de la desaparición de nitrato y la disminución de la desnitrificación, como se ha reportado previamente en experimentos in situ (Hall, 1984). Por otra parte, el acetileno que se agregó durante los experimentos (15\% $\mathrm{v} / \mathrm{v})$, no inhibió la comunidad desnitrificante, a pesar de que dicha concentración se ha usado con efectividad en muestras acuáticas (Abe, et al., 2000, Beaulieu, et al., 2010, Wang, et al., 2012, Baxter, et al., 2012). Esto probablemente se debe a los bajos niveles de nitrato en el agua, lo cual se ha reportado como un factor negativo para la acción del acetileno, sin embargo, otros estudios reportan que algunas muestras han requerido más de $290 \mu \mathrm{M}(>0,7$ $\mathrm{KPa})$ y hasta $0,3 \mathrm{mM}$ de acetileno $(30 \mathrm{ml})$ para que se produzca la inhibición total de la comunidad desnitrificante (Mosier \& Francis, 2010), mientras que con solo $29 \mu \mathrm{M}$ ya se inhibe la comunidad anammox y la nitrificante.

En el futuro deben desarrollarse estudios sobre las variaciones espacio-temporales (diarias, mensuales e interanuales) de las emisiones netas y los flujos de $\mathrm{N}_{2} \mathrm{O}$, así como de los procesos microbianos involucrados en el reciclaje de ambos gases invernadero, que permitan determinar la importancia del aporte natural y antropogénico de la hidroeléctrica de Prado a las emisiones globales de gases invernadero.

\section{Conclusiones}

En general, con esta investigación se determinó que la nitrificación contribuye activamente a la producción de $\mathrm{N}_{2} \mathrm{O}$ en el epilimnio del embalse de Prado y que en comparación con otras hidroeléctricas y lagos en el mundo, las concentraciones de $\mathrm{N}_{2} \mathrm{O}$ y $\mathrm{CH}_{4}$ cuantificadas en la columna de agua fueron moderadamente altas. Sin embargo, la sobresaturación estimada para ambos gases en las aguas superficiales y profundas sugiere que el embalse podría estar liberando cantidades significativas de estos gases invernadero a la atmósfera.

\section{Agradecimientos}

A la Universidad del Tolima y a su oficina de investigaciones y desarrollo científico, por la financiación de esta investigación y a A. Castro y M. Pacheco, por su colaboración en el trabajo de campo.

\section{Conflicto de intereses}

Los autores declaran que no tienen ningún conflicto de intereses.

\section{Bibliografía}

Abe, D.S, Kato, K., Adams, D.D., Terai, H., Tundisi, J.G. (2000). Contribution of free-living and attached bacteria to denitrification in the hipolimnion of a mesotrophic Japanese lake. Microbes and Environments. 15 (2): 93-101.

Barros, N, Cole, J.J., Tranvik, L.J., Prairie, Y.T., Bastviken, D., Huszar, V.L.M., del Giorgio, P., Roland, F. (2011). Carbon emission from hydroelectric reservoirs linked to reservoir age and latitude. Nature Geoscience. 4: 593-596.

Baxter, A.M., Johnson, L., Edgerton, J., Royer, T., Lef, L.G. (2012). Structure and function of denitrifying bacterial assemblages in low-order Indiana streams. Freshwater Science. 31 (2): 304-317.

Beaulieu, J.J, Tank, J.L., Hamilton, S.K., Wollheims, W.M., Hall, R.O., Mulholland, P.J., Peterson, B.J., Ashkenas, L.R., Cooperi, L.W., Dahmj, C.N., Doddsk, W.K., Grimml, N.B., Johnsonm, S.L., McDowelln, W.H., Pooleo, G.C., Valettp, H.M., Arangoq,n C.P., Bernotr, M.J., Burgins, A.J., Crenshawj, C.L., Heltont, A.M., Johnsonu, L.T., O•Brienv, J.M., Pottern, J.D., Sheibleyl, R.W., Sobotaw, D.J., Thomasg, S.M. (2010). Nitrous oxide emission from denitrification in stream and river networks. PNAS. 1: 1-6.

Borrel, G, Jézéquel, D., Biderr-Petit, C., Morel-Desrosiers, N., Morel, J-P., Peyret, P., Fonty, G., Lehours, A-C. 2011. Production and consumption of methane in freshwater lake ecosystems. Research in Microbiology. 162: 832-847.

Bruner, R.C. \& García-Gil, L.J. (1996). Sulfide-induced dissimilatory nitrate reduction to ammonia in anaerobic freshwater sediments. FEMS Microbiology and Ecology. 21: 131-38.

Burgin, A.J. \& Hamilton, S.K. (2007). Have we overemphasized the role of denitrification in aquatic ecosystems? A review of nitrate removal pathways. Frontiers in Ecology and the Environment. 5 (2): 89-96.

Casciotti, K.L. \& Buchwald, C. (2012). Insights on the marine microbial nitrogen cycle from isotopic approaches to nitrification. Frontiers in Microbiology. 3 (356): 1-14.

Castro-González M. (2014). Evaluación de la comunidad desnitrificante de tipo nos $Z$ en la columna de agua de Isla del Sol, embalse de Prado, Tolima. Revista de la Academia Colombiana de Ciencias Exactas, Físicas y Naturales. 38 (149): 385-92. 
Castro-González, M. \& Pacheco, M. (2014). Diversity of nosZtype denitrifiers in a tropical hydroelectric reservoir in the south east of Colombia. Abstracts 15th International symposium on microbial ecology-ISME-15.

Deemer, B.R., Harrison, J.A., Whitling, E.W. (2011). Microbial dinitrogen and nitrous oxide production in a small eutrophic reservoir: An in situ approach to quantifying hypolimnetic process rates. Limnology and Oceanography. 56 (4): 11891199.

Demarty, M. \& Bastien, J. (2011). GHG emissions from hydroelectric reservoirs in tropical and equatorial regions: Review of 20 years of $\mathrm{CH}_{4}$ emission measurements. Energy Policy. 39 (7): 4197-4206.

Deutzman, J.S. \& Schink, B. (2011). Anaerobic oxidation of methane in sediments of Lake Constance, an oligotrophic freshwater lake. Applied and Environmental Microbiology. 77: 4429-4436.

US Environmental Protection Agency. (2010). Methane and Nitrous Oxide Emissions from Natural Sources. Washington, DC, $330 \mathrm{p}$.

Fearnside, P.M. (2005). Do hydroelectric dams mitigate global warming? The case of Brazil's Curná-Una dam. Mitigation and Adaptation Strategies to Global Change. 10 (4): 675-691.

Figueiredo, V, Santoro, A.L., van Weerelt, M., Enrich-Prast, A. (2012). Sediment denitrification, DNRA and anammox rates in tropical floodplain lake (Pantanal, Brazil). Oecologia Australis. 16 (4): 734-744.

Furlanetto, L.M, Marinho, C.C., Palma-Silva, C., Albertoni, E.F., Figueiredo-Barros, M.P., de Assis, F. (2012). Methane levels in shallow subtropical lake sediments: Dependence on the trophic status of the lake and allochthonous input. Limnologica. 42: 151-155.

Ginestet, P., Audic, J.M., Urbain, V., Block, J.C. (1998). Estimation of nitrifying bacterial activities by measuring oxygen uptake in the presence of the metabolic inhibitors allylthiourea and azide. Applied and Environmental Microbiology. 64: 2266-2268.

Goldenfum, J.A. (2012). Challenges and solutions for assessing the impact of freshwater reservoir on natural GHG emissions. Ecohydrology and Hydrobiology. 12 (2): 115-122.

Grossart, H-P, Frindte, K., Dziallas. C., Eckert, W., Tang, K.W. (2011). ZZCycles. 18 (3): GB3022. http://dx.doi. org/:10.1029/2003GB002197

Speksnijder, A.G.C.L, Kowalchuk, G.A., Roest, K., Laanbroek, H.J. (1998). Recovery of a Nitrosomonas-like 16S rDNA sequence group from freshwater habitats. Systematic and Applied Microbiology. 21: 321-30.
St. Louis, V, Kelly, L.C.A., Duchemin, E., Rudd, J.W.M., Rosenberg, D.M. (2000). Reservoir surfaces as sources of greenhouse gases to the atmosphere: A global estimate. BioScience. 50: 766-775.

Stocker, T.F, Qin, D., Plattner, G-K., Tignor, M., Allen, S.K., Boschung, J., Nauels, Y.X., Bex, V., Midgley, P.M (Editors). (2013). The Physical Science Basis. Contributions of Working Group I to the Fifth Assessment Report of the Intergovernmental Panel on Climate Change. Cambridge, United Kingdom and New York, NY, USA: Cambridge University Press. 1535 p.

Sturm, K, Yuan, Z., Gibbes, B., Grinham, A. (2013). Methane and nitrous oxide sources and emissions in a subtropical freshwater reservoir, south east Queensland, Australia. Biogeosciences Discussions. 10: 19485-19508.

Wang, H.J., Wang, W.D., Yin, C.Q., Wang, Y.C., Lu, J.W. (2006). Littoral zones as the "hotspots" of nitrous oxide emission in a hyper-eutrophic lake in China. Atmospheric Environment. 40: 5522-5527.

Wang, S.L, Liu, C.Q., Yeager, K.M., Wan, C.J., Li, J., Tao, F.X., Lue, Y.C., Liu, F., Fan, C.X. (2009). The spatial distribution and emission of nitrous oxide in a large eutrophic lake in eastern China: Anthropogenic effects. Science of the Total Environment. 407: 3330-3337.

Wang, S, Yeager, K.M., Wan, G., Liu, C., Tao, F., Fan, C. 2010. Short-term field observations of nitrous oxide saturations in lake Taihu, China: The need for high temporal resolution studies. Journal of Environmental Quality. 39: 1858-1863.

Wang, C, Zhu, G., Wang, Y., Wang, S., Yin, Ch. (2012). Nitrous oxide reductase gene (nosZ) and $\mathrm{N}_{2} \mathrm{O}$ reduction along the littoral gradient of a eutrophic freshwater lake. Journal of Environmental Science. 25 (1): 44-52.

Weiss, R.F. \& Price, B.A. (1980). Nitrous oxide solubility in water and seawater. Marine Chemistry. 8: 347-359.

Wiesenburg, D.A. \& Guinasso, N.L. (1979). Equilibrium solubilities of methane, carbon monoxide, and hydrogen in water and sea water. Journal of Chemical and Engineering Data. 24 (4): 356-360.

Wrage, N., Velthof, G.L., Oenema, O., Laanbroek, H.J. (2004). Acetylene and oxygen as inhibitor of nitrous oxide production in Nitrosomonas europaea and Nitrospira briensis: A cautionary tale. FEMS Microbiology and Ecology. 47: 13-18.

Yang, L, Lu, F., Zhou, X., Wang, X., Duan, X., Sun, B. (2014). Progress in the studies on the greenhouse gas emissions from reservoirs. Acta Ecologica Sinica. 34: 204-212. 